\title{
B-spline approximations of the Gaussian, their Gabor frame properties, and approximately dual frames
}

\author{
Ole Christensen, Hong Oh Kim, Rae Young Kim
}

August 17, 2017

\begin{abstract}
We prove that Gabor systems generated by certain scaled B-splines can be considered as perturbations of the Gabor systems generated by the Gaussian, with a deviation within an arbitrary small tolerance whenever the order $N$ of the B-spline is sufficiently large. As a consequence we show that for any choice of translation/modulation parameters $a, b>0$ with $a b<1$, the scaled version of $B_{N}$ generates Gabor frames for $N$ sufficiently large. Considering the Gabor frame decomposition generated by the Gaussian and a dual window, the results lead to estimates of the deviation from perfect reconstruction that arise when the Gaussian is replaced by a scaled B-spline, or when the dual window of the Gaussian is replaced by certain explicitly given and compactly supported linear combinations of the B-splines. In particular, this leads to a family of approximate dual windows of a very simple form, leading to "almost perfect reconstruction" within any desired error tolerance whenever the product $a b$ is sufficiently small. In contrast, the known (exact) dual windows have a very complicated form. A similar analysis is sketched with the scaled B-splines replaced by certain truncations of the Gaussian. As a consequence of the approach we prove (mostly known) convergence results for the considered scaled B-splines to the Gaussian in the

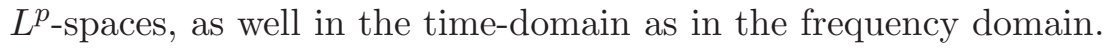

Keywords: Gaussian, B-splines, frames, dual frames

2010 Mathematics Subject Classifications: 42C40, 42C15

\section{Introduction}

For any parameters $a, b \in \mathbb{R}$, define the translation operator $T_{a}$ and modulation operators $E_{b}$ acting on $L^{2}(\mathbb{R})$ by $T_{a} f(x)=f(x-a), E_{b} f(x)=e^{2 \pi i b x} f(x), x \in \mathbb{R}$. It is well-known (see [13, 14, 15]) that for any $\alpha>0$, the Gabor system $\left\{E_{m b} T_{n a} \varphi\right\}_{m, n \in \mathbb{Z}}$ generated by the Gaussian $\varphi(x)=e^{-\alpha x^{2}}$ forms a frame for all $a, b>0$ such that $a b<1$. The choice of the Gaussian is well motivated within time-frequency analysis due to the fast decay of the Gaussian and its Fourier transform. In this paper we consider approximations of the Gaussian via functions with compact support, namely, scaled B-splines and truncated 
Gaussians. We choose to consider the (centered) B-splines $B_{N}, N \in \mathbb{N}$, defined recursively by

$$
B_{1}(x)=\chi_{[-1 / 2,1 / 2]}(x), \quad B_{N+1}(x)=B_{N} * B_{1}(x)=\int_{-1 / 2}^{1 / 2} B_{N}(x-t) d t .
$$

The case of the scaled B-splines is clearly connected to the central limit theorem; it is also motivated by results by Unser et al. [16], who proved that certain scalings of the $N$ th order B-splines $B_{N}$ converge (pointwise and in certain $L^{p}$-spaces) to the Gaussian when $N \rightarrow \infty$. Generalizations are obtained in the papers by Bricks [1] and Goh et al. [9]. We show that for any $a, b>0$ such that $a b<1$, the mentioned functions generate Gabor frames whenever the order of the B-spline is sufficiently high or the truncation of the Gaussian has sufficiently large support. These results are interesting for applications where compact support of the window is desired.

As a consequence of the above results we are able to construct explicitly given approximately dual frames for Gabor frames $\left\{E_{m b} T_{n a} \varphi\right\}_{m, n \in \mathbb{Z}}$ generated by the Gaussian, for certain choices of the parameters $a, b>0$. The approximate dual windows have an extremely simple and explicit form: they are constant on large intervals, have compact support, and are given explicitly as certain linear combinations of integer-shifts of the Bsplines $B_{N}$ for some fixed $N \in \mathbb{N}$. Furthermore, by choosing the parameter $N$ sufficiently high, we can get as close to perfect reconstruction as desired (see (1.2) and the subsequent text for details about this terminology). In contrast, the known (exact) dual windows of the Gaussian have a very complicated form; see [10, 11].

The approach in the current paper leads to proofs of certain other convergence results. Indeed, we prove that the considered scaled B-splines converge to the Gaussian in all the $L^{p}$-spaces for $p \in[1, \infty]$; this result was also obtained in [9], while [16] considered the case $p \in[2, \infty]$. We also prove that the Fourier transform of the considered scaled $B_{N}$ converge uniformly to the Gaussian when $N \rightarrow \infty$.

In the rest of this introduction we set the stage by collecting some definitions and necessary results from frame theory. In Section 2 we then prove the mentioned frame results for the scaled B-splines. In Section 3 a similar analysis is sketched for truncated Gaussians. In Section 4 the results are applied to construct explicitly given approximately dual frames associated with certain Gabor frames generated by the Gaussian. The consequences about convergence of the scaled B-splines and its Fourier transform in $L^{p}$-spaces are collected in Section 5 .

A sequence $\left\{f_{k}\right\}_{k \in I}$ in a separable Hilbert space $\mathcal{H}$ is called a frame if there exist constants $A, B>0$ such that

$$
A\|f\|^{2} \leq \sum_{k \in I}\left|\left\langle f, f_{k}\right\rangle\right|^{2} \leq B\|f\|^{2}, \forall f \in \mathcal{H}
$$

Thus, frames generalize the concept of an orthonormal basis. The key property of frames is that they lead to unconditionally convergent series expansions of the elements in the underlying Hilbert space. Indeed, if $\left\{f_{k}\right\}_{k \in I}$ is a frame for $\mathcal{H}$, there always exist a frame 
$\left\{g_{k}\right\}_{k \in I}$ such that

$$
f=\sum_{k \in I}\left\langle f, g_{k}\right\rangle f_{k}=\sum_{k \in I}\left\langle f, f_{k}\right\rangle g_{k}
$$

A frame $\left\{g_{k}\right\}_{k \in I}$ satisfying (1.2) is called a dual frame of $\left\{f_{k}\right\}_{k \in I}$; and (1.2) is expressed by saying that the frames $\left\{f_{k}\right\}_{k \in I}$ and $\left\{g_{k}\right\}_{k \in I}$ lead to perfect reconstruction. Note also that a sequence $\left\{f_{k}\right\}_{k \in I}$ satisfying at least the upper condition in (1.1) is called a Bessel sequence with Bessel bound B.

An alternative formulation can be given in terms of operator theory. If the sequence $\left\{f_{k}\right\}_{k \in I}$ satisfies at least the upper frame condition, one defines the synthesis operator $T$ by

$$
T: \ell^{2}(I) \rightarrow \mathcal{H}, T\left\{c_{k}\right\}_{k \in I}:=\sum_{k \in I} c_{k} f_{k}
$$

It is well-known that $T$ is well-defined and bounded. Denoting the synthesis operator for the sequence $\left\{g_{k}\right\}_{k \in I}$ by $U$, perfect reconstruction is equivalent with $T U^{*}=I$.

A weaker concept was introduced in [7]. We say that two Bessel sequences $\left\{f_{k}\right\}_{k \in I}$ and $\left\{g_{k}\right\}_{k \in I}$ form approximately dual frames if $\left\|I-T U^{*}\right\|<1$; or, in other words, if there exists a constant $\mu<1$ such that

$$
\left\|f-\sum_{k \in I}\left\langle f, f_{k}\right\rangle g_{k}\right\| \leq \mu\|f\|, \forall f \in \mathcal{H}
$$

The rationale behind approximately dual frames is that all kinds of implementations involve certain imprecisions; thus, as long as we can control the deviation from perfect reconstruction measured by the parameter $\mu$ in (1.3), approximately dual frames can in practice be as good as exact dual frames.

Most of the results in the paper will be based on perturbation results, in particular, the result stated below. The result stated in (i) is classic [5]; we include a short proof of (ii).

Lemma 1.1 Let $\left\{f_{k}\right\}_{k \in I}$ be a frame for a separable Hilbert space $\mathcal{H}$ with bounds $A, B$, and let $\left\{\widetilde{f}_{k}\right\}_{k \in I}$ be a sequence in $\mathcal{H}$. Then the following hold:

(i) If there exists a constant $R<A$ such that

$$
\sum_{k \in I}\left|\left\langle f, f_{k}-\widetilde{f}_{k}\right\rangle\right|^{2} \leq R\|f\|^{2}, \forall f \in \mathcal{H}
$$

then $\left\{\tilde{f}_{k}\right\}_{k \in I}$ is a frame with bounds $A\left(1-\sqrt{\frac{R}{A}}\right)^{2}, B\left(1+\sqrt{\frac{R}{B}}\right)^{2}$.

(ii) Assume that $\left\{g_{k}\right\}_{k \in I}$ is a dual frame of $\left\{f_{k}\right\}_{k \in I}$, with upper frame bound $B_{g}$, and that

$$
\left\|\sum_{k \in I} c_{k}\left(f_{k}-\tilde{f}_{k}\right)\right\| \leq \mu\left(\sum_{k \in I}\left|c_{k}\right|^{2}\right)^{1 / 2}
$$


for some $\mu \geq 0$ and all finite sequences $\left\{c_{k}\right\}_{k \in I}$. Then

$$
\left\|f-\sum_{k \in I}\left\langle f, \widetilde{f}_{k}\right\rangle g_{k}\right\| \leq \mu \sqrt{B_{g}}\|f\|, \forall f \in \mathcal{H} .
$$

In particular $\left\{g_{k}\right\}_{k \in I}$ and $\left\{\widetilde{f}_{k}\right\}_{k \in I}$ are approximately dual frames if $\mu \sqrt{B_{g}}<1$.

Proof of (ii): It is well-known that if (1.5) holds for all finite sequences, then it holds for all sequences in $\ell^{2}(I)$; also, (1.5) is just a different way of expressing that $\left\{f_{k}-\widetilde{f}_{k}\right\}_{k \in I}$ is a Bessel sequence with bound at most $\mu^{2}$. Letting $c_{k}=\left\langle f, f_{k}\right\rangle$ then leads to

$$
\begin{aligned}
\left\|f-\sum_{k \in I}\left\langle f, \widetilde{f}_{k}\right\rangle g_{k}\right\| & =\left\|\sum_{k \in I}\left\langle f, f_{k}\right\rangle g_{k}-\sum_{k \in I}\left\langle f, \widetilde{f}_{k}\right\rangle g_{k}\right\| \\
& \leq \sqrt{B_{g}}\left(\sum_{k \in I}\left|\left\langle f, f_{k}-\widetilde{f}_{k}\right\rangle\right|^{2}\right)^{1 / 2} \\
& \leq \mu \sqrt{B_{g}}|| f \|, \forall f \in \mathcal{H},
\end{aligned}
$$

as desired.

In words, Lemma 1.1 (i) says that we can check that a sequence $\left\{\widetilde{f}_{k}\right\}_{k \in I}$ is a frame by proving that it is "close" to a frame $\left\{f_{k}\right\}_{k \in I}$, in the sense that $\left\{f_{k}-\widetilde{f}_{k}\right\}_{k \in I}$ is a Bessel sequence with a sufficiently small bound. The importance of Lemma 1.1 (ii) lies in the fact that it measures the deviation from perfect reconstruction which occur when the frame $\left\{f_{k}\right\}_{k \in I}$ is perturbed.

For Gabor systems, the Bessel bound is typically calculated via the so-called CCcondition (see [2] or [4], Theorem 11.4.2), stated in Proposition 1.2 (i) below. Via standard manipulations the CC-condition can also be formulated in the Fourier domain; since we also need this version of the condition, this is stated in Proposition 1.2 (ii). We define the Fourier transform of $f \in L^{1}(\mathbb{R})$ by

$$
\widehat{f}(\gamma)=\int_{-\infty}^{\infty} f(x) e^{-2 \pi i \gamma x} d x
$$

with the usual extension to $L^{2}(\mathbb{R})$.

Proposition 1.2 Let $g \in L^{2}(\mathbb{R}), a, b>0$. Then the following hold:

(i) If

$$
B(g, a, b):=\frac{1}{b} \sup _{x \in[0, a]} \sum_{k \in \mathbb{Z}}\left|\sum_{n \in \mathbb{Z}} g(x-n a) \overline{g(x-n a-k / b)}\right|<\infty,
$$

then $\left\{E_{m b} T_{n a} g\right\}_{m, n \in \mathbb{Z}}$ is a Bessel sequence with Bessel bound $B(g, a, b)$.

(ii) If

$$
\widehat{B}(g, a, b):=\frac{1}{a} \sup _{\gamma \in[0, b]} \sum_{k \in \mathbb{Z}}\left|\sum_{n \in \mathbb{Z}} \widehat{g}(\gamma-n b) \overline{\widehat{g}(\gamma-n b-k / a)}\right|<\infty,
$$

then $\left\{E_{m b} T_{n a} g\right\}_{m, n \in \mathbb{Z}}$ is a Bessel sequence with Bessel bound $\widehat{B}(g, a, b)$. 


\section{Approximation of the Gaussian via B-splines}

Our first goal is to show that for any choice of the translation/modulation parameters, the Bessel bound of the Gabor system generated by the difference between certain scaled B-splines and the Gaussian tend to zero as $N \rightarrow \infty$. Eventually this leads to the main conclusion in this section, namely, that for any $a, b>0$ with $a b<1$, the scaled B-spline $B_{N}$ generate Gabor frames whenever the order $N$ is sufficiently large.

In order to estimate the difference between a Gaussian and the scaled B-splines pointwise, we will need the following result concerning the pointwise difference between a Gaussian and the $N$ th power of the sinc-function. We will state two versions: a precise pointwise estimate, and a qualitative estimate, concerning the behavior as $N \rightarrow \infty$. Given a function $f: \mathbb{N} \rightarrow \mathbb{C}$ the notation $f(N)=o(1)$ means that for every $\epsilon>0$, there exists $N_{0} \in \mathbb{N}$ such that for $N \geq N_{0},|f(N)| \leq \epsilon$. Even when several parameters are involved like in (2.1) below, the symbol $o(1)$ will always refer to the dependence on the parameter $N$.

Lemma 2.1 Let $N \geq 14$ be an integer. Then

$$
\left|e^{-\frac{x^{2} N}{6}}-\left(\frac{\sin x}{x}\right)^{N}\right| \leq \begin{cases}\frac{4}{5 e^{2} N}\left(1+\frac{17 \ln N}{7 N}\right)=\frac{4}{5 e^{2} N}(1+o(1)), & |x|<\sqrt{\frac{12 \ln N}{N}} \\ \frac{4(\ln N)^{2}}{5 N^{3}}\left(1+\frac{17 \ln N}{7 N}\right)=\frac{4(\ln N)^{2}}{5 N^{3}}(1+o(1)), & \sqrt{\frac{12 \ln N}{N}} \leq|x| \leq \frac{\pi}{2}\end{cases}
$$

Proof. Fix $N \geq 14$. By symmetry it is enough to consider $x \in\left[0, \frac{\pi}{2}\right]$. A crucial step in the proof is to observe that

$$
e^{-\frac{x^{2}}{6}} \geq \frac{\sin x}{x}, \forall x \in\left[0, \frac{\pi}{2}\right]
$$

so let us argue for this first. Taylor's theorem implies that

$$
\frac{\sin x}{x} \leq 1-\frac{x^{2}}{3 !}+\frac{x^{4}}{5 !} \text { and } e^{-\frac{x^{2}}{6}} \geq 1-\left(\frac{x^{2}}{6}\right)+\frac{1}{2 !}\left(\frac{x^{2}}{6}\right)^{2}-\frac{1}{3 !}\left(\frac{x^{2}}{6}\right)^{3} .
$$

A direct calculation shows that $1-\left(\frac{x^{2}}{6}\right)+\frac{1}{2 !}\left(\frac{x^{2}}{6}\right)^{2}-\frac{1}{3 !}\left(\frac{x^{2}}{6}\right)^{3} \geq 1-\frac{x^{2}}{3 !}+\frac{x^{4}}{5 !}$. Thus we obtain that (2.2) holds for $x \in\left[0, \frac{\pi}{2}\right]$, as desired.

Now we will use that

$$
\left|e^{-\frac{x^{2} N}{6}}-\left(\frac{\sin x}{x}\right)^{N}\right|=e^{-\frac{x^{2} N}{6}}\left|1-\left(\frac{\sin x}{x} e^{\frac{x^{2}}{6}}\right)^{N}\right|
$$

Fix $x \in\left[0, \frac{\pi}{2}\right]$. Taylor's theorem again implies that $\frac{\sin x}{x} \geq 1-\frac{x^{2}}{3 !}+\frac{x^{4}}{5 !}-\frac{x^{6}}{7 !}$ and $e^{\frac{x^{2}}{6}} \geq$ $1+\frac{x^{2}}{6}+\frac{x^{4}}{72}$. In the following calculation we will use that $\frac{x^{8}}{12096}-\frac{x^{10}}{362880}=\frac{x^{8}}{362880}\left(30-x^{2}\right)>0$. 
Indeed, this implies that

$$
\begin{aligned}
\left(\frac{\sin x}{x} e^{\frac{x^{2}}{6}}\right)^{N} & \geq\left(1-\frac{x^{2}}{3 !}+\frac{x^{4}}{5 !}-\frac{x^{6}}{7 !}\right)^{N}\left(1+\frac{x^{2}}{6}+\frac{x^{4}}{72}\right)^{N} \\
& =\left(1-\frac{x^{4}}{180}-\frac{17 x^{6}}{15120}+\frac{x^{8}}{12096}-\frac{x^{10}}{362880}\right)^{N} \\
& \geq\left(1-\frac{x^{4}}{180}-\frac{17 x^{6}}{15120}\right)^{N} .
\end{aligned}
$$

Using the inequality $(1-t)^{N} \geq 1-N t$ for $0<t<1$ and that $\frac{x^{4}}{180}+\frac{17 x^{6}}{15120}<1$, we have $\left(\frac{\sin x}{x} e^{\frac{x^{2}}{6}}\right)^{N} \geq 1-N\left(\frac{x^{4}}{180}+\frac{17 x^{6}}{15120}\right)=1-\frac{N x^{4}}{180}\left(1+\frac{17}{84} x^{2}\right)$. This together with (2.2) implies that $0 \leq 1-\left(\frac{\sin x}{x} e^{\frac{x^{2}}{6}}\right)^{N} \leq \frac{N x^{4}}{180}\left(1+\frac{17}{84} x^{2}\right)$. Therefore we have

$$
\left|e^{-\frac{x^{2} N}{6}}-\left(\frac{\sin x}{x}\right)^{N}\right| \leq e^{-\frac{x^{2} N}{6}} \frac{N x^{4}}{180}\left(1+\frac{17}{84} x^{2}\right)=: f_{N}(x),
$$

by (2.3). Note that $\sqrt{\frac{12 \ln N}{N}} \leq \frac{\pi}{2}$ for $N \geq 14$. We now split $\left[0, \frac{\pi}{2}\right]$ into two intervals $\left[0, \sqrt{\frac{12 \ln N}{N}}\left[\right.\right.$ and $\left[\sqrt{\frac{12 \ln N}{N}}, \frac{\pi}{2}\right]$ :

(1) For $x \in\left[0, \sqrt{\frac{12 \ln N}{N}}[,(2.4)\right.$ implies that

$$
\left|e^{-\frac{x^{2} N}{6}}-\left(\frac{\sin x}{x}\right)^{N}\right| \leq e^{-\frac{x^{2} N}{6}} \frac{N x^{4}}{180}\left(1+\frac{17 \ln N}{7 N}\right) .
$$

Fixing $N \in \mathbb{N}$, the function $x \mapsto e^{-\frac{x^{2} N}{6}} \frac{N x^{4}}{180}$ attains its maximum value $\frac{4}{5 e^{2} N}$ on the interval $\left[0, \sqrt{\frac{12 \ln N}{N}}\left[\right.\right.$ at $x=\sqrt{\frac{12}{N}} ;$ thus

$$
\left|e^{-\frac{x^{2} N}{6}}-\left(\frac{\sin x}{x}\right)^{N}\right| \leq \frac{4}{5 e^{2} N}\left(1+\frac{17 \ln N}{7 N}\right)=\frac{4}{5 e^{2} N}(1+o(1)) .
$$

(2) Note that for $N \geq 14$, the function $f_{N}$ is decreasing on the interval $\left[\sqrt{\frac{12 \ln N}{N}}, \frac{\pi}{2}\right]$. Thus, for $x \in\left[\sqrt{\frac{12 \ln N}{N}}, \frac{\pi}{2}\right]$,

$$
f_{N}(x) \leq f_{N}\left(\sqrt{\frac{12 \ln N}{N}}\right)=\frac{4(\ln N)^{2}}{5 N^{3}}\left(1+\frac{17 \ln N}{7 N}\right)=\frac{4(\ln N)^{2}}{5 N^{3}}(1+o(1)),
$$

as desired.

In the next lemma we introduce the exact scaling of the B-splines that we use throughout the paper; it goes back to the paper [16] by Unser et al. The result describes the asymptotic behavior of these functions. 
Lemma 2.2 Let $N \in \mathbb{N}$ and let

$$
p_{N}(x):=\frac{1}{\sqrt{2 \pi}} e^{-x^{2} / 2}-\sqrt{\frac{N}{12}} B_{N}\left(\sqrt{\frac{N}{12}} x\right) .
$$

Given any $a, b>0$, the Bessel bound $\left.\widehat{B} p_{N}, a, b\right)$ in (1.8) can be estimated by

$$
\widehat{B}\left(p_{N}, a, b\right) \leq \frac{64 \ln N}{b 25 \pi^{2} e^{4} N^{2}}(1+o(1)) .
$$

In particular, for any $\epsilon>0$, there exists a positive integer $N(a, b)$ such that for $N \geq N(a, b)$ the Gabor system $\left\{E_{m b} T_{n a} p_{N}\right\}_{m, n \in \mathbb{Z}}$ is a Bessel sequence with bound at most $\epsilon$.

Proof. Let $N \geq 14$ be an integer. Using that $\widehat{B_{N}}(\gamma)=\left(\frac{\sin \pi \gamma}{\pi \gamma}\right)^{N}$, a change of variable yields that

$$
\widehat{p_{N}}(\gamma)=e^{-2 \pi^{2} \gamma^{2}}-\left(\frac{\sin \left(\pi \sqrt{\frac{12}{N}} \gamma\right)}{\pi \sqrt{\frac{12}{N}} \gamma}\right)^{N}
$$

By letting $x=\pi \sqrt{\frac{12}{N}} \gamma$, Lemma 2.1 implies that

$$
\left|e^{-2 \pi^{2} \gamma^{2}}-\left(\frac{\sin \left(\pi \sqrt{\frac{12}{N}} \gamma\right)}{\pi \sqrt{\frac{12}{N}} \gamma}\right)^{N}\right| \leq \begin{cases}\frac{4}{5 e^{2} N}\left(1+\frac{17 \ln N}{7 N}\right), & |\gamma|<\frac{\sqrt{\ln N}}{\pi} \\ \frac{4(\ln N)^{2}}{5 N^{3}}\left(1+\frac{17 \ln N}{7 N}\right), & \frac{\sqrt{\ln N}}{\pi} \leq|\gamma| \leq \frac{\sqrt{N}}{4 \sqrt{3}}\end{cases}
$$

Also, clearly

$$
\left|e^{-2 \pi^{2} \gamma^{2}}-\left(\frac{\sin \left(\pi \sqrt{\frac{12}{N}} \gamma\right)}{\pi \sqrt{\frac{12}{N}} \gamma}\right)^{N}\right| \leq e^{-2 \pi^{2} \gamma^{2}}+\left|\frac{1}{\pi \sqrt{\frac{12}{N}} \gamma}\right|^{N}, \gamma \in \mathbb{R}
$$

We now estimate the Bessel bound (1.8) for $g=p_{N}$. We first note that

$$
\begin{aligned}
\widehat{B}\left(p_{N}, a, b\right) & \leq \frac{1}{a} \sup _{\gamma \in[0, b]} \sum_{k \in \mathbb{Z}} \sum_{n \in \mathbb{Z}}\left|\widehat{p_{N}}(\gamma-n b) \widehat{p_{N}}(\gamma-n b-k / a)\right| \\
& \leq \frac{1}{a}\left(\sup _{\gamma \in[0, b], n \in \mathbb{Z}} \sum_{k \in \mathbb{Z}}\left|\widehat{p_{N}}(\gamma-n b-k / a)\right|\right)\left(\sup _{\gamma \in[0, b]} \sum_{n \in \mathbb{Z}}\left|\widehat{p_{N}}(\gamma-n b)\right|\right)
\end{aligned}
$$

Estimate of $\sum_{k \in \mathbb{Z}}\left|\widehat{p_{N}}(\gamma-n b-k / a)\right|:$ For a fixed $n \in \mathbb{Z}, \gamma-n b-k / a$ hits the interval ] $-\frac{\sqrt{\ln N}}{\pi}, \frac{\sqrt{\ln N}}{\pi}\left[\right.$ for at most $\left\lceil\frac{2 a \sqrt{\ln N}}{\pi}\right\rceil$ values of $k \in \mathbb{Z}$ and its contribution is at most $\left\lceil\frac{2 a \sqrt{\ln N}}{\pi}\right\rceil \frac{4}{5 e^{2} N}\left(1+\frac{17 \ln N}{7 N}\right)$ by (2.8) . Also, by (2.8) $)$, the contribution from $\gamma-n b-k / a$ hitting 
the interval $\left.]-\frac{\sqrt{N}}{4 \sqrt{3}},-\frac{\sqrt{\ln N}}{\pi}\right] \cup\left[\frac{\sqrt{\ln N}}{\pi}, \frac{\sqrt{N}}{4 \sqrt{3}}\left[\right.\right.$ is at most $2\left\lceil a\left(\frac{\sqrt{N}}{4 \sqrt{3}}-\frac{\sqrt{\ln N}}{\pi}\right)\right\rceil \frac{4(\ln N)^{2}}{5 N^{3}}\left(1+\frac{17 \ln N}{7 N}\right)$. By (2.9), the contribution from $\gamma-n b-k / a$ hitting the interval $\left[\frac{\sqrt{N}}{4 \sqrt{3}}, \infty[\right.$ is at most

$$
\begin{aligned}
& \sum_{k=0}^{\infty}\left|\widehat{p_{N}}\left(\frac{\sqrt{N}}{4 \sqrt{3}}+\frac{k}{a}\right)\right| \leq \sum_{k=0}^{\infty} e^{-2 \pi^{2}\left(\frac{\sqrt{N}}{4 \sqrt{3}}+\frac{k}{a}\right)^{2}}+\sum_{k=0}^{\infty}\left|\frac{\sqrt{N}}{\sqrt{12} \pi\left(\frac{\sqrt{N}}{4 \sqrt{3}}+\frac{k}{a}\right)}\right|^{N} \\
&=: \quad(I)+(I I)
\end{aligned}
$$

We now estimate (I) and (II) as follows:

$$
(I) \leq e^{-\frac{\pi^{2} N}{24}} \sum_{k=0}^{\infty} e^{-\left(\frac{\pi^{2} \sqrt{N}}{\sqrt{3} a}\right) k}=\frac{e^{-\frac{\pi^{2} N}{24}}}{1-e^{-\frac{\pi^{2} \sqrt{N}}{\sqrt{3} a}}}
$$

and

$$
\begin{aligned}
(I I) & =\sum_{k=0}^{\infty}\left|\frac{\sqrt{N}}{\sqrt{12} \pi \frac{\sqrt{N}}{4 \sqrt{3}}\left(1+\frac{4 \sqrt{3} k}{a \sqrt{N}}\right)}\right|^{N} \\
& =\left(\frac{2}{\pi}\right)^{N} \sum_{k=0}^{\infty}\left(1+\frac{4 \sqrt{3} k}{a \sqrt{N}}\right)^{-N} \\
& \leq\left(\frac{2}{\pi}\right)^{N}\left(1+\int_{0}^{\infty}\left(1+\frac{4 \sqrt{3} x}{a \sqrt{N}}\right)^{-N} d x\right) \\
& =\left(\frac{2}{\pi}\right)^{N}\left(1+\frac{a \sqrt{N}}{4 \sqrt{3}(N-1)}\right) .
\end{aligned}
$$

The contribution from $\left.]-\infty,-\frac{\sqrt{N}}{4 \sqrt{3}}\right]$ is at most the same as above. Therefore,

$$
\begin{aligned}
\sum_{k \in \mathbb{Z}}\left|\widehat{p_{N}}(\gamma-n b-k / a)\right| \leq & \left(1+\frac{2 a \sqrt{\ln N}}{\pi}\right) \frac{4}{5 e^{2} N}\left(1+\frac{17 \ln N}{7 N}\right) \\
& +\left(2+2 a\left(\frac{\sqrt{N}}{4 \sqrt{3}}-\frac{\sqrt{\ln N}}{\pi}\right)\right) \frac{4(\ln N)^{2}}{5 N^{3}}\left(1+\frac{17 \ln N}{7 N}\right) \\
& +2\left(\frac{e^{-\frac{\pi^{2} N}{24}}}{1-e^{-\frac{\pi^{2} \sqrt{N}}{\sqrt{3} a}}}+\left(\frac{2}{\pi}\right)^{N}\left(1+\frac{a \sqrt{N}}{4 \sqrt{3}(N-1)}\right)\right) \\
=: & P_{N}(a) \\
= & \frac{8 a \sqrt{\ln N}}{5 \pi e^{2} N}(1+o(1)) .
\end{aligned}
$$

Note that the above estimate is independent on the choice of $n \in \mathbb{Z}$ and $b>0$. 
Estimate of $\sum_{n \in \mathbb{Z}}\left|\widehat{p_{N}}(\gamma-n b)\right|:$ Using that the estimate (2.12) also holds in the particular case where the parameter $a$ takes the value $1 / b$, it follows that

$$
\begin{aligned}
\sum_{n \in \mathbb{Z}}\left|\widehat{p_{N}}(\gamma-n b)\right| \leq & \left(1+\frac{2 \sqrt{\ln N}}{b \pi}\right) \frac{4}{5 e^{2} N}\left(1+\frac{17 \ln N}{7 N}\right) \\
& +\left(2+\frac{2}{b}\left(\frac{\sqrt{N}}{4 \sqrt{3}}-\frac{\sqrt{\ln N}}{\pi}\right)\right) \frac{4(\ln N)^{2}}{5 N^{3}}\left(1+\frac{17 \ln N}{7 N}\right) \\
& +2\left(\frac{e^{-\frac{\pi^{2} N}{24}}}{1-e^{-\frac{\pi^{2} b \sqrt{N}}{\sqrt{3}}}}+\left(\frac{2}{\pi}\right)^{N}\left(1+\frac{\sqrt{N}}{4 b \sqrt{3}(N-1)}\right)\right) \\
= & P_{N}(1 / b)=\frac{8 \sqrt{\ln N}}{b 5 \pi e^{2} N}(1+o(1)) .
\end{aligned}
$$

Hence (2.10) implies that the Bessel bound of $\left\{E_{m b} T_{n a} p_{N}\right\}_{m, n \in \mathbb{Z}}$ can be estimated by

$$
\widehat{B}\left(p_{N}, a, b\right) \leq \frac{P_{N}(a) P_{N}(1 / b)}{a}=\frac{64 \ln N}{b 25 \pi^{2} e^{4} N^{2}}(1+o(1)) .
$$

It is now clear that $\widehat{B}\left(p_{N}, a, b\right) \rightarrow 0$ as $N \rightarrow \infty$.

Lemma 2.2 now allows us to give an easy proof for the main result in this section, namely, that for any $a, b>0$ with $a b<1$, the considered scaled B-splines $B_{N}$ generate Gabor frames whenever the order $N$ is sufficiently high.

Theorem 2.3 For any $a, b>0$ such that $a b<1$, there exists a positive integer $N(a, b)$ such that the function

$$
g_{N}(x):=\sqrt{\frac{N}{12}} B_{N}\left(\sqrt{\frac{N}{12}} x\right)
$$

generates a Gabor frame $\left\{E_{m b} T_{n a} g_{N}\right\}_{m, n \in \mathbb{Z}}$ whenever $N \geq N(a, b)$.

Proof. We have already mentioned that the Gaussian generates a Gabor frame for any $a, b>0$ such that $a b<1$. Fixing such $a, b$, let $A$ denote a lower frame bound for the Gabor frame generated by the function $\frac{1}{\sqrt{2 \pi}} e^{-x^{2} / 2}$. By Lemma 2.2 we can choose an integer $N(a, b)$ such that the Bessel bound $\widehat{B}\left(p_{N}, a, b\right)$ for the function $p_{N}$ in (2.5) is smaller than $A$ for $N \geq N(a, b)$. By Lemma 1.1 this implies that the function $g_{N}$ in (2.14) generates a Gabor frame for $N \geq N(a, b)$.

Note that the frame properties of the (non-scaled) B-splines is a very active research area. We will not directly use any of the results from the literature, so we just refer to the recent paper [12] and the extensive list of references therein for more information.

The proof of Theorem 2.3 gives some information about how to choose the integer $N(a, b)$ : it should be chosen such that the Bessel bound $\widehat{B}\left(p_{N}, a, b\right)$ for the function $p_{N}$ 
in (2.5) is smaller than the lower frame bound $A$ for the Gabor frame generated by the Gaussian. The next result, which is a consequence of the calculations in the proof of Lemma 2.2. gives more explicit information about how to choose $N(a, b)$ such that the Bessel bound for the function $p_{N}$ does not exceed a prescribed maximal value:

Corollary 2.4 Let $N_{0} \geq 14$ be an integer, and let

$$
\begin{aligned}
K(x)= & \left(\frac{4}{5 e^{2}}\left(\frac{2 x}{\pi}+\frac{1}{\sqrt{\ln N_{0}}}\right)+\left(\frac{\ln N_{0}}{N_{0}}\right)^{3 / 2} \frac{4}{5}\left(\frac{2}{\sqrt{N_{0}}}+\frac{x}{2 \sqrt{3}}\right)\right)\left(1+\frac{17 \ln N_{0}}{7 N_{0}}\right) \\
& +\frac{2 N_{0}}{\sqrt{\ln N_{0}}}\left(\frac{e^{-\frac{\pi^{2} N_{0}}{24}}}{1-e^{-\frac{\pi^{2} \sqrt{N_{0}}}{\sqrt{3} x}}}+\left(\frac{2}{\pi}\right)^{N_{0}}\left(1+\frac{x \sqrt{N_{0}}}{4 \sqrt{3}\left(N_{0}-1\right)}\right)\right) .
\end{aligned}
$$

Define $p_{N}$ as in (2.5). Given any $\epsilon>0$, choose

$$
N(a, b):=\left\lfloor\frac{K(a) K(1 / b)}{a \epsilon}\right\rfloor+N_{0} .
$$

Then $\widehat{B}\left(p_{N(a, b)}, a, b\right)<\epsilon$.

Proof. First, fix any integer $N_{0} \geq 14$. It is easy to show by induction that for $\alpha \leq \frac{14}{15}$ and $N \geq N_{0}$, we have that $\alpha^{N} \leq \frac{\alpha^{N_{0} N_{0}}}{N}$. Since $e^{-\pi^{2} / 24} \leq \frac{14}{15}$ and $2 / \pi \leq \frac{14}{15}$ we can now use this to conclude that

$$
e^{-\frac{\pi^{2} N}{24}} \leq e^{-\frac{\pi^{2} N_{0}}{24}} \frac{N_{0}}{N} \text { and }\left(\frac{2}{\pi}\right)^{N} \leq\left(\frac{2}{\pi}\right)^{N_{0}} \frac{N_{0}}{N} .
$$

Moreover, the functions $N \mapsto \frac{\ln N}{N}, N \mapsto e^{-\frac{\pi^{2} \sqrt{N}}{a \sqrt{3}}}, N \mapsto \frac{\sqrt{N}}{N-1}$ are decreasing. Thus, with the given definition of $K(x)$ and $P_{N}$ defined by (2.12),

$$
\begin{aligned}
P_{N}(a) \leq & \frac{\sqrt{\ln N}}{N}\left(\frac{4}{5 e^{2}}\left(\frac{2 a}{\pi}+\frac{1}{\sqrt{\ln N}}\right)\left(1+\frac{17 \ln N}{7 N}\right)\right. \\
& +\left(\frac{\ln N}{N}\right)^{3 / 2} \frac{4}{5}\left(\frac{2}{\sqrt{N}}+\frac{a}{2 \sqrt{3}}\right)\left(1+\frac{17 \ln N}{7 N}\right) \\
& \left.+2 \frac{N}{\sqrt{\ln N}}\left(\frac{e^{-\frac{\pi^{2} N}{24}}}{1-e^{-\frac{\pi^{2} \sqrt{N}}{\sqrt{3} a}}}+\left(\frac{2}{\pi}\right)^{N}\left(1+\frac{a \sqrt{N}}{4 \sqrt{3}(N-1)}\right)\right)\right) \\
\leq & \frac{\sqrt{\ln N}}{N} K(a) .
\end{aligned}
$$

This together with (2.13) implies that

$$
\widehat{B}\left(p_{N}, a, b\right) \leq \frac{K(a) K(1 / b) \ln N}{a N^{2}} \leq \frac{K(a) K(1 / b)}{a N} .
$$

Note that we have that $\frac{K(a) K(1 / b)}{a \epsilon}<N(a, b)$ by (2.16). Thus $\widehat{B}\left(p_{N(a, b)}, a, b\right) \leq \frac{K(a) K(1 / b)}{a N(a, b)}<\epsilon$, as desired. 


\section{Frame properties of truncated Gaussians}

In this section we will repeat the procedure of Section 2 with the scaled B-splines replaced by certain compactly supported "truncations" of the Gaussian, namely, functions of the form

$$
\varphi_{N}(x):=(g(x)-g(N)) \chi_{[-N, N]}(x)
$$

for some $N \in \mathbb{N}$ and with $g(x)=e^{-x^{2} / 2}$. Note that the subtraction of the value $g(N)$ implies that $\varphi_{N}$ is continuous. Due to the fast decay of the exponential function $g$, the function $\varphi_{N}$ will in general provide a very good approximation of $g$ for large values of $N$. In the next lemma we show that the truncated versions of the Gaussian approximate the Gaussian well in terms of the Bessel condition, whenever $N$ is large. Similar considerations can of course be done for other functions, but for the sake of the flow of the current paper we stick to the Gaussian.

Lemma 3.1 Let $N \in \mathbb{N}$. Consider

$$
q_{N}(x):=e^{-x^{2} / 2}-\left(e^{-x^{2} / 2}-e^{-N^{2} / 2}\right) \chi_{[-N, N]}(x) .
$$

Given any $a, b>0$, the Bessel bound $B\left(q_{N}, a, b\right)$ in (1.7) can be estimated by

$$
\begin{aligned}
B\left(q_{N}, a, b\right) & \leq\left(1+2 b N+\frac{2}{1-e^{-\frac{N}{b}}}\right)\left(1+\frac{2 N}{a}+\frac{2}{1-e^{-a N}}\right) \frac{e^{-N^{2}}}{b} \\
& =\frac{4 N^{2} e^{-N^{2}}}{a}(1+o(1)) .
\end{aligned}
$$

In particular, for any $\epsilon>0$, there exists a positive integer $N(a, b)$ such that for $N \geq N(a, b)$ the Gabor system $\left\{E_{m b} T_{n a} q_{N}\right\}_{m, n \in \mathbb{Z}}$ is a Bessel sequence with bound at most $\epsilon$.

Proof. The proof is similar to the proof of Lemma 2.2, so we only sketch it. We estimate the Bessel bound (1.7) for $g=q_{N}$. We note that

$$
B\left(q_{N}, a, b\right) \leq \frac{1}{b}\left(\sup _{x \in[0, a], n \in \mathbb{Z}} \sum_{k \in \mathbb{Z}}\left|q_{N}(x-n a-k / b)\right|\right)\left(\sup _{x \in[0, a]} \sum_{n \in \mathbb{Z}}\left|q_{N}(x-n a)\right|\right) .
$$

We also note that

$$
q_{N}(x)= \begin{cases}e^{-N^{2} / 2}, & x \in[-N, N] \\ e^{-x^{2} / 2}, & x \in[-N, N]^{c}\end{cases}
$$

Estimate of $\sum_{k \in \mathbb{Z}}\left|q_{N}(x-n a-k / b)\right|:$ For a fixed $n \in \mathbb{Z}, x-n a-k / b$ hits $]-N, N[$ at most $\lceil 2 b N\rceil$ times for $k$ 's and its contribution is at most $\lceil 2 b N\rceil e^{-N^{2} / 2}$ by (3.3). By (3.3) again, the contribution from $x-n a-k / b$ hitting the interval $[N, \infty[$ is at most

$$
\sum_{k=0}^{\infty}\left|q_{N}\left(N+\frac{k}{b}\right)\right| \leq \sum_{k=0}^{\infty} e^{-\frac{N^{2}}{2}-\frac{N k}{b}}=\frac{e^{-\frac{N^{2}}{2}}}{1-e^{-\frac{N}{b}}}
$$


The contribution from ] $-\infty,-N]$ is at most the same as above. Therefore,

$$
\sum_{k \in \mathbb{Z}}\left|q_{N}(x-n a-k / b)\right| \leq(1+2 b N) e^{-\frac{N^{2}}{2}}+\frac{2 e^{-\frac{N^{2}}{2}}}{1-e^{-\frac{N}{b}}}=: Q_{N}(b)=2 b N e^{-\frac{N^{2}}{2}}(1+o(1)) .
$$

Estimate of $\sum_{n \in \mathbb{Z}}\left|q_{N}(x-n a)\right|:$ Applying the above result with $b=1 / a$ yields that

$$
\sum_{n \in \mathbb{Z}}\left|q_{N}(x-n a)\right| \leq\left(1+\frac{2 N}{a}\right) e^{-\frac{N^{2}}{2}}+\frac{2 e^{-\frac{N^{2}}{2}}}{1-e^{-a N}}=Q_{N}(1 / a)=\frac{2 N e^{-\frac{N^{2}}{2}}}{a}(1+o(1)) .
$$

Hence (3.2) together with (3.4) and (3.5) implies that the Bessel bound of $\left\{E_{m b} T_{n a} q_{N}\right\}_{m, n \in \mathbb{Z}}$ can be estimated by

$$
\begin{aligned}
B\left(q_{N}, a, b\right) & \leq \frac{Q_{N}(b) Q_{N}(1 / a)}{b} \\
& =\left(1+2 b N+\frac{2}{1-e^{-\frac{N}{b}}}\right)\left(1+\frac{2 N}{a}+\frac{2}{1-e^{-a N}}\right) \frac{e^{-N^{2}}}{b} \\
& =\frac{4 N^{2} e^{-N^{2}}}{a}(1+o(1)) .
\end{aligned}
$$

This clearly implies that $B\left(q_{N}, a, b\right) \rightarrow 0$ as $N \rightarrow \infty$, as desired.

As in Theorem 2.3, Lemma 3.1 has the following consequence:

Theorem 3.2 For any $a, b>0$ such that $a b<1$, there exists a positive integer $N(a, b)$ such that the function

$$
\varphi_{N}(x):=\left(e^{-x^{2} / 2}-e^{-N^{2} / 2}\right) \chi_{[-N, N]}(x)
$$

generates a Gabor frame $\left\{E_{m b} T_{n a} \varphi_{N}\right\}_{m, n \in \mathbb{Z}}$ whenever $N \geq N(a, b)$.

Analog to the case of B-spline approximations in Section 2 one can derive a quantitative estimate on the number $N(a, b)$ in Theorem $\underline{3.2}$, we leave the details to the interested reader.

\section{Approximately dual frames}

The known dual windows associated with the Gabor frames generated by the Gaussian are quite complicated (see the end of the section for a more detailed discussion). On the other hand, for sufficiently small modulation parameters $b>0$ and the translation parameter $a=1$, B-spline generated Gabor frames $\left\{E_{m b} T_{n} B_{N}\right\} m, n \in \mathbb{Z}$ have convenient dual windows that are finite linear combinations of integer-translates of the B-spline [3, 6]. In this section we prove that replacing the exact dual window of the Gaussian by these simple dual windows of the B-spline can get us as close to perfect reconstruction as desired. 


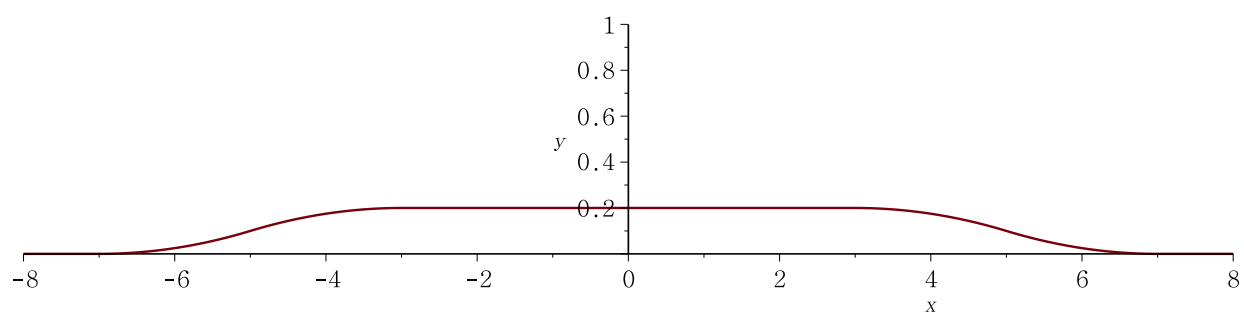

Figure 1: The dual window $h_{N}$ in Proposition 4.1 for $N=3$.

The starting point is the observation that for certain choices of translation/modulation parameters one can construct a dual frame associated with the Gabor frames generated by the considered scaled B-splines; see [3, 6] for details.

Proposition 4.1 Let $N \in \mathbb{N}$. Let $a:=\sqrt{\frac{12}{N}}$ and $b:=\frac{1}{2 N-1} \sqrt{\frac{N}{12}}$. Then the functions

$$
g_{N}(x):=\sqrt{\frac{N}{12}} B_{N}\left(\sqrt{\frac{N}{12}} x\right)
$$

and

$$
h_{N}(x):=b \sqrt{\frac{12}{N}} \sum_{n=-N+1}^{N-1} B_{N}\left(\sqrt{\frac{N}{12}} x+n\right)
$$

generate a pair of dual frames $\left\{E_{m b} T_{n a} g_{N}\right\}_{m, n \in \mathbb{Z}}$ and $\left\{E_{m b} T_{n a} h_{N}\right\}_{m, n \in \mathbb{Z}}$.

Note that in Proposition 4.1 we have $a b=(2 N-1)^{-1}$; thus, for large values of $N \in \mathbb{N}$ the constructed Gabor frames are highly redundant. Figure 1 shows the dual window $h_{N}$ for $N=3$. The shape of $h_{3}$ is typical for all the functions $h_{N}, N \in \mathbb{N}$; indeed, $h_{N}$ is constant on the interval $[-\sqrt{3 N}, \sqrt{3 N}]$ and supported on the interval $\left[-\left(\frac{3 N}{2}-1\right) \sqrt{\frac{12}{N}},\left(\frac{3 N}{2}-1\right) \sqrt{\frac{12}{N}}\right]$.

Lemma 4.2 Let $N \in \mathbb{N}$. Let $a:=\sqrt{\frac{12}{N}}$ and $b:=\frac{1}{2 N-1} \sqrt{\frac{N}{12}}$. Define $h_{N}$ as in (4.2). Then the Bessel bound $B\left(h_{N}, a, b\right)$ in (1.7) for $\left\{E_{m b} T_{n a} h_{N}\right\}_{m, n \in \mathbb{Z}}$ can be estimated by

$$
B\left(h_{N}, a, b\right) \leq \frac{9 \sqrt{3}}{2 \sqrt{N}}(1+o(1))
$$

Proof. We estimate the Bessel bound (1.7) for $g=h_{N}$ with $a=\sqrt{\frac{12}{N}}$ and $b=\frac{1}{2 N-1} \sqrt{\frac{N}{12}}$. As we already saw in (3.2),

$$
B\left(h_{N}, a, b\right) \leq \frac{1}{b}\left(\sup _{x \in[0, a], n \in \mathbb{Z}} \sum_{k \in \mathbb{Z}}\left|h_{N}(x-n a-k / b)\right|\right)\left(\sup _{x \in[0, a]} \sum_{n \in \mathbb{Z}}\left|h_{N}(x-n a)\right|\right) .
$$


Since $B_{N}$ satisfies the partition of unity property, it follows directly from our knowledge of the support of $h_{N}$ that

$$
h_{N}(x) \leq b \sqrt{\frac{12}{N}} \chi_{\left[-\left(\frac{3 N}{2}-1\right) \sqrt{\frac{12}{N}},\left(\frac{3 N}{2}-1\right) \sqrt{\frac{12}{N}}\right]}(x) .
$$

By arguments similar to the ones used in the proof of Lemma 3.1, we have

$$
\sum_{k \in \mathbb{Z}}\left|h_{N}(x-n a-k / b)\right| \leq\left(1+2 b\left(\frac{3 N}{2}-1\right) \sqrt{\frac{12}{N}}\right) b \sqrt{\frac{12}{N}}
$$

and

$$
\sum_{n \in \mathbb{Z}}\left|h_{N}(x-n a)\right| \leq\left(1+\frac{2}{a}\left(\frac{3 N}{2}-1\right) \sqrt{\frac{12}{N}}\right) b \sqrt{\frac{12}{N}} .
$$

By (4.4) and direct calculation based on the above estimates and the given values of $a, b$, the Bessel bound of $\left\{E_{m b} T_{n a} h_{N}\right\}_{m, n \in \mathbb{Z}}$ can be estimated by

$$
B\left(h_{N}, \sqrt{\frac{12}{N}}, \frac{1}{2 N-1} \sqrt{\frac{N}{12}}\right) \leq \frac{9 \sqrt{3}}{2 \sqrt{N}}(1+o(1)),
$$

as desired.

The next result proves that for $N \in \mathbb{N}$ sufficiently large and certain choices of $a, b>0$, the Gabor system $\left\{E_{m b} T_{n a} h_{N}\right\}_{m, n \in \mathbb{Z}}$ is an approximately dual frame of a Gabor system generated by a (multiple of a) Gaussian:

Theorem 4.3 Let $N \in \mathbb{N}$, and let $a:=\sqrt{\frac{12}{N}}$ and $b:=\frac{1}{2 N-1} \sqrt{\frac{N}{12}}$. Define $h_{N}$ as in (4.2) and let

$$
\widetilde{g}(x)=\frac{1}{\sqrt{2 \pi}} e^{-x^{2} / 2} .
$$

Then

$$
\left\|f-\sum_{m, n \in \mathbb{Z}}\left\langle f, E_{m b} T_{n a} \widetilde{g}\right\rangle E_{m b} T_{n a} h_{N}\right\| \leq \frac{24 \sqrt{6 \ln N}}{5 \pi e^{2} N}(1+o(1))\|f\|, \forall f \in L^{2}(\mathbb{R}) .
$$

Proof. The idea of the proof is to apply Lemma 1.1 (ii) on the dual frames

$$
\left\{f_{k}\right\}_{k \in I}:=\left\{E_{m b} T_{n a} g_{N}\right\}_{m, n \in \mathbb{Z}},\left\{g_{k}\right\}_{k \in I}:=\left\{E_{m b} T_{n a} h_{N}\right\}_{m, n \in \mathbb{Z}} .
$$

Letting $\left\{\widetilde{f}_{k}\right\}_{k \in I}:=\left\{E_{m b} T_{n a} \widetilde{g}\right\}_{m, n \in \mathbb{Z}}$, the condition (1.5) is satisfied with $\mu^{2}=\widehat{B}\left(p_{N}, a, b\right)$, where $p_{N}$ is the function defined in (2.5) and $\widehat{B}\left(p_{N}, a, b\right)$ is given by (1.8). Thus, the term $\mu \sqrt{B_{g}}$ in (1.6) corresponds precisely to

$$
\left(\widehat{B}\left(p_{N}, a, b\right) B\left(h_{N}, a, b\right)\right)^{1 / 2} .
$$


In order to estimate $\widehat{B}\left(p_{N}, a, b\right)$ we will use some of the calculations from the proof of Lemma 2.2, Fix any integer $N \geq 14$. Applying (2.12) with $a:=\sqrt{\frac{12}{N}}$ yields that

$$
\begin{aligned}
P_{N}(a)= & P_{N}\left(\sqrt{\frac{12}{N}}\right) \\
= & \left(1+2 \sqrt{\frac{12}{N}} \frac{\sqrt{\ln N}}{\pi}\right) \frac{4}{5 e^{2} N}\left(1+\frac{17 \ln N}{7 N}\right) \\
& +\left(2+2 \sqrt{\frac{12}{N}}\left(\frac{\sqrt{N}}{4 \sqrt{3}}-\frac{\sqrt{\ln N}}{\pi}\right)\right) \frac{4(\ln N)^{2}}{5 N^{3}}\left(1+\frac{17 \ln N}{7 N}\right) \\
& +2\left(\frac{e^{-\frac{\pi^{2} N}{24}}}{1-e^{-\frac{\pi^{2} N}{6}}}+\left(\frac{2}{\pi}\right)^{N}\left(1+\frac{1}{2(N-1)}\right)\right) \\
= & \frac{16 \sqrt{3} \sqrt{\ln N}}{5 \pi e^{2} N \sqrt{N}}(1+o(1)) .
\end{aligned}
$$

Similarly, we have

$$
P_{N}(1 / b)=P_{N}\left((2 N-1) \sqrt{\frac{12}{N}}\right)=\frac{32 \sqrt{3} \sqrt{\ln N}}{5 \pi e^{2} \sqrt{N}}(1+o(1)) .
$$

Inserting this into (2.13) yields

$$
\widehat{B}\left(p_{N}, a, b\right) \leq \frac{P_{N}(a) P_{N}(1 / b)}{a}=\sqrt{\frac{N}{12}} P(a) P(1 / b)=\frac{2^{8} \sqrt{3} \ln N}{5^{2} \pi^{2} e^{4} N \sqrt{N}}(1+o(1)) .
$$

This together with (4.3) implies that

$$
\widehat{B}\left(p_{N}, a, b\right) B\left(h_{N}, a, b\right) \leq \frac{2^{7} 3^{3} \ln N}{5^{2} \pi^{2} e^{4} N^{2}}(1+o(1)) .
$$

Now (4.5) is a consequence of Lemma 1.1 (ii).

Theorem 4.3 has a clear potential for applications, as we will explain in detail now. Indeed, the known (exact) dual windows associated with the Gabor frame generated by the Gaussian have a very complicated structure. To the best of our knowledge, the only explicit expressions for dual windows associated with the Gaussian were obtained by Janssen [10, 11. Consider for the moment the Gaussian on the form $g(x)=2^{1 / 4} e^{-\pi x^{2}}$, and fix $a, b>0$ such that $a b<1$. Furthermore, let

$$
K:=\sum_{k \in \mathbb{Z}}(-1)^{k}(2 k+1) e^{-\pi a(k+1 / 2)^{2}}, \quad \operatorname{erfc}(x):=\frac{2}{\sqrt{\pi}} \int_{x}^{\infty} e^{-s^{2}} d s .
$$


Then Janssen showed in [10] that for any $\epsilon>0, \epsilon<1-a b$, the function

$$
\widetilde{g}_{\epsilon}(x):=2^{-1 / 4} b K^{-1} e^{\pi x^{2}} \sum_{k \in \mathbb{Z}}(-1)^{k} e^{-\pi a(k+1 / 2)^{2} / b} \operatorname{erfc}[(x-(k+1 / 2) a) \sqrt{\pi / \epsilon}]
$$

generates a dual frame of $\left\{E_{m b} T_{n a} g\right\}_{m, n \in \mathbb{Z}}$. An explicit dual window is calculated in [11 for the case where $(a b)^{-1}$ is an even integer (the calculations are more involved when $(a b)^{-1}$ is an odd integer). On the other hand, as already described the functions $h_{N}$ in (4.2) have an extremely simple and explicit structure. Theorem 4.3 shows that for certain sufficiently small values of the product $a b$ we can get arbitrary close to perfect reconstruction by replacing the exact dual window by the function $h_{N}$ for a sufficiently large value of $N \in \mathbb{N}$. For example, doing so with a tolerance that is smaller then the "machine precision" (in a slightly informal language) will be as good as having perfect reconstruction.

Note that in Theorem 4.3 each choice of $N \in \mathbb{N}$ leads to new and fixed values for the translation parameter $a$ and the modulation parameter $b$, given by typically irrational numbers. For general frames this can be problematic, due to inevitable roundoff errors that will occur in practice. However, the Gaussian and the (scaled) B-splines $B_{N}, N \geq 2$, belong to the Feichtinger algebra $\mathcal{S}_{0}$, which implies that the Bessel bound for the Gabor system $\left\{E_{m b} T_{n a} h_{N}\right\}_{m, n \in \mathbb{Z}}$ depends continuously on the parameters $a, b>0$, cf. the results

by Feichtinger and Kaiblinger [8]. Thus, Theorem 4.3 is less sensitive towards roundoff errors than one could think at a first glance.

\section{Convergence in $L^{p}$-spaces}

In this section we derive some easy consequences of our approach in Section 2. In Theorem 5.2 we prove that the considered scalings of the B-splines converge to the Gaussian in $L^{p}(\mathbb{R})$ for $p \in[1, \infty]$; this was also obtained by Goh et al. in [9], while Unser et al. [16] proved it for $p \in[2, \infty]$. First we prove that the Fourier transform of the scaled B-splines converge to the Gaussian in $L^{q}(\mathbb{R})$ for $q \in[1, \infty]$; the range $q \in[1, \infty[$ was first considered by Unser et al. [16].

Theorem 5.1 Let $q \in[1, \infty]$. Then

$$
\mathcal{F}\left(\sqrt{\frac{N}{12}} B_{N}\left(\sqrt{\frac{N}{12}} \cdot\right)\right)(\gamma)=\left(\frac{\sin \left(\pi \sqrt{\frac{12}{N}} \gamma\right)}{\pi \sqrt{\frac{12}{N}} \gamma}\right)^{N} \rightarrow e^{-2 \pi^{2} \gamma^{2}}
$$

in $L^{q}(\mathbb{R})$ as $N \rightarrow \infty$.

Proof. Fix any integer $N \geq 14$. We will distinguish between the cases $q=\infty$ and $q \in[1, \infty[$.

(1) The case $q=\infty$ : By (2.9), we see that

$$
\left|e^{-2 \pi^{2} \gamma^{2}}-\left(\frac{\sin \left(\pi \sqrt{\frac{12}{N}} \gamma\right)}{\pi \sqrt{\frac{12}{N}} \gamma}\right)^{N}\right| \leq e^{-\frac{\pi^{2} N}{24}}+\left(\frac{2}{\pi}\right)^{N},|\gamma| \geq \frac{\sqrt{N}}{4 \sqrt{3}} .
$$


This together with (2.8) implies that

$$
\sup _{\gamma \in \mathbb{R}}\left|e^{-2 \pi^{2} \gamma^{2}}-\left(\frac{\sin \left(\pi \sqrt{\frac{12}{N}} \gamma\right)}{\pi \sqrt{\frac{12}{N}} \gamma}\right)^{N}\right| \leq \max \left\{\frac{4}{5 e^{2} N}\left(1+\frac{17 \ln N}{7 N}\right), e^{-\frac{\pi^{2} N}{24}}+\left(\frac{2}{\pi}\right)^{N}\right\} \rightarrow 0
$$

as $N \rightarrow \infty$.

(2) The case $q \in[1, \infty[$ : By (2.8) and (2.9) , we have

$$
\begin{aligned}
& \int_{-\infty}^{\infty}\left|e^{-2 \pi^{2} \gamma^{2}}-\left(\frac{\sin \left(\pi \sqrt{\frac{12}{N}} \gamma\right)}{\pi \sqrt{\frac{12}{N}} \gamma}\right)^{N}\right|^{q} d \gamma \\
& \leq \int_{|\gamma|<\frac{\sqrt{\ln N}}{\pi}}\left(\frac{4}{5 e^{2} N}\left(1+\frac{17 \ln N}{7 N}\right)\right)^{q} d \gamma+\int_{\frac{\sqrt{\ln N}}{\pi} \leq|\gamma| \leq \frac{\sqrt{N}}{4 \sqrt{3}}}\left(\frac{4(\ln N)^{2}}{5 N^{3}}\left(1+\frac{17 \ln N}{7 N}\right)\right)^{q} d \gamma \\
& +\int_{|\gamma| \geq \frac{\sqrt{N}}{4 \sqrt{3}}}\left(e^{-2 \pi^{2} \gamma^{2}}+\left|\frac{1}{\pi \sqrt{\frac{12}{N}} \gamma}\right|^{N}\right)^{q} d \gamma
\end{aligned}
$$

Using that $(a+b)^{q} \leq \max \left\{(2 a)^{q},(2 b)^{q}\right\} \leq 2^{q}\left(a^{q}+b^{q}\right)$, we have

$$
\begin{aligned}
& \int_{|\gamma| \geq \frac{\sqrt{N}}{4 \sqrt{3}}}\left(e^{-2 \pi^{2} \gamma^{2}}+\left|\frac{1}{\pi \sqrt{\frac{12}{N}} \gamma}\right|^{N}\right)^{q} d \gamma \\
& \leq 2^{q}\left(\int_{|\gamma| \geq \frac{\sqrt{N}}{4 \sqrt{3}}} e^{-2 \pi^{2} \gamma^{2} q} d \gamma+\int_{|\gamma| \geq \frac{\sqrt{N}}{4 \sqrt{3}}}\left|\frac{1}{\pi \sqrt{\frac{12}{N}} \gamma}\right|^{N q} d \gamma\right) .
\end{aligned}
$$

Direct calculations show that

$$
\begin{aligned}
& \int_{|\gamma| \geq \frac{\sqrt{N}}{4 \sqrt{3}}} e^{-2 \pi^{2} \gamma^{2} q} d \gamma \leq 2 \int_{\frac{\sqrt{N}}{4 \sqrt{3}}}^{\infty} e^{-2 \pi^{2} \gamma q} d \gamma=\frac{e^{-\left(2 \pi^{2} q \frac{\sqrt{N}}{4 \sqrt{3}}\right)}}{\pi^{2} q}\left(\text { if } \frac{\sqrt{N}}{4 \sqrt{3}} \geq 1\right) \\
& \int_{|\gamma| \geq \frac{\sqrt{N}}{4 \sqrt{3}}}\left|\frac{1}{\pi \sqrt{\frac{12}{N}} \gamma}\right|^{N q} d \gamma=\left(\frac{2}{\pi}\right)^{N q} \frac{\sqrt{N}}{2 \sqrt{3}(N q-1)}(\text { if } N q>1) .
\end{aligned}
$$


Hence

$$
\begin{aligned}
& \int_{-\infty}^{\infty}\left|e^{-2 \pi^{2} \gamma^{2}}-\left(\frac{\sin \left(\pi \sqrt{\frac{12}{N}} \gamma\right)}{\pi \sqrt{\frac{12}{N}} \gamma}\right)^{N}\right|^{q} d \gamma \\
& \leq 2\left(\left(\frac{4}{5 e^{2} N}\right)^{q} \frac{\sqrt{\ln N}}{\pi}+\left(\frac{4(\ln N)^{2}}{5 N^{3}}\right)^{q}\left(\frac{\sqrt{N}}{4 \sqrt{3}}-\frac{\sqrt{\ln N}}{\pi}\right)\right)\left(1+\frac{17 \ln N}{7 N}\right)^{q} \\
& +2^{q}\left(\frac{\left.e^{-\left(2 \pi^{2} q \frac{\sqrt{N}}{4 \sqrt{3}}\right.}\right)}{\pi^{2} q}+\left(\frac{2}{\pi}\right)^{N q} \frac{\sqrt{N}}{2 \sqrt{3}(N q-1)}\right) \\
& \leq C_{q} \frac{\sqrt{\ln N}}{N^{q}}
\end{aligned}
$$

for some $C_{q}>0$, and therefore tends to zero as $N \rightarrow \infty$.

We are now ready to prove that the considered scaled versions of the B-splines $B_{N}$ converge to the Gaussian in $L^{p}(\mathbb{R}), p \in[1, \infty]$ as $N \rightarrow \infty$.

Theorem 5.2 Let $p \in[1, \infty]$. Then

$$
\sqrt{\frac{N}{12}} B_{N}\left(\sqrt{\frac{N}{12}} x\right) \rightarrow \frac{1}{\sqrt{2 \pi}} e^{-\frac{x^{2}}{2}}
$$

in $L^{p}(\mathbb{R})$ as $N \rightarrow \infty$.

Proof. We will distinguish between the cases $p=\infty$ and $p \in[1, \infty[$.

(1) The case $p=\infty$ : Using the function $p_{N}$ in (2.5) and its Fourier transform in (2.7), the inequality $\left\|p_{N}\right\|_{\infty} \leq\left\|\widehat{p}_{N}\right\|_{L^{1}(\mathbb{R})}$ and (5.1) with $q=1$ imply that

$$
\begin{aligned}
\left\|\frac{1}{\sqrt{2 \pi}} e^{-\frac{x^{2}}{2}}-\sqrt{\frac{N}{12}} B_{N}\left(\sqrt{\frac{N}{12}} x\right)\right\|_{\infty} & \leq \int_{-\infty}^{\infty}\left|e^{-2 \pi^{2} \gamma^{2}}-\left(\frac{\sin \left(\pi \sqrt{\frac{12}{N}} \gamma\right)}{\pi \sqrt{\frac{12}{N}} \gamma}\right)^{N}\right| d \gamma \\
& \leq C_{1} \frac{\sqrt{\ln N}}{N}
\end{aligned}
$$

which tends to zero as $N \rightarrow \infty$.

(2) The case $p \in\left[1, \infty\left[:\right.\right.$ Since $\operatorname{supp} B_{N}\left(\sqrt{\frac{N}{12}} \cdot\right)=[-\sqrt{3 N}, \sqrt{3 N}]$, we see that

$$
\begin{aligned}
& \int_{-\infty}^{\infty}\left|\frac{1}{\sqrt{2 \pi}} e^{-\frac{x^{2}}{2}}-\sqrt{\frac{N}{12}} B_{N}\left(\sqrt{\frac{N}{12}} x\right)\right|^{p} d x \\
& \leq \int_{|x| \leq \sqrt{3 N}}\left|\frac{1}{\sqrt{2 \pi}} e^{-\frac{x^{2}}{2}}-\sqrt{\frac{N}{12}} B_{N}\left(\sqrt{\frac{N}{12}} x\right)\right|^{p} d x+\int_{|x| \geq \sqrt{3 N}}\left|\frac{1}{\sqrt{2 \pi}} e^{-\frac{x^{2}}{2}}\right|^{p} d x .
\end{aligned}
$$


By (5.2), we have

$$
\int_{|x| \leq \sqrt{3 N}}\left|\frac{1}{\sqrt{2 \pi}} e^{-\frac{x^{2}}{2}}-\sqrt{\frac{N}{12}} B_{N}\left(\sqrt{\frac{N}{12}} x\right)\right|^{p} d x \leq 2 \sqrt{3 N}\left(\frac{C_{1} \sqrt{\ln N}}{N}\right)^{p}=\frac{2 C_{1}^{p} \sqrt{3}(\sqrt{\ln N})^{p}}{N^{p-\frac{1}{2}}} .
$$

Also direct calculations show

$$
\int_{|x| \geq \sqrt{3 N}}\left|\frac{1}{\sqrt{2 \pi}} e^{-\frac{x^{2}}{2}}\right|^{p} d x \leq \int_{|x| \geq \sqrt{3 N}}\left|\frac{1}{\sqrt{2 \pi}} e^{-\frac{x}{2}}\right|^{p} d x=\frac{4}{p}\left(\frac{1}{\sqrt{2 \pi}}\right)^{p} e^{-\frac{p \sqrt{3 N}}{2}} .
$$

Hence

$\int_{-\infty}^{\infty}\left|\sqrt{\frac{N}{12}} B_{N}\left(\sqrt{\frac{N}{12}} x\right)-\frac{1}{\sqrt{2 \pi}} e^{-\frac{x^{2}}{2}}\right|^{p} d x \leq \frac{2 C_{1}^{p} \sqrt{3}(\sqrt{\ln N})^{p}}{N^{p-\frac{1}{2}}}+\frac{4}{p}\left(\frac{1}{\sqrt{2 \pi}}\right)^{p} e^{-\frac{p \sqrt{3 N}}{2}} \rightarrow 0$

as $N \rightarrow \infty$.

Consider again the function $g_{N}$ in (2.14). As a consequence of our results we obtain that a certain scaled version of the Fourier transform of $g_{N}$ "behave very similar as $g_{N}$ " as $N \rightarrow \infty$. Recall that for $a>0$ the scaling operator $D_{a}$ on $L^{2}(\mathbb{R})$ is defined by $D_{a} f(x)=$ $a^{-1 / 2} f\left(a^{-1} x\right)$. The scaling operator is unitary.

Corollary 5.3 Let $p \in[1, \infty]$. Then $g_{N}-D_{2 \pi} \mathcal{F} g_{N} \rightarrow 0$ in $L^{p}(\mathbb{R})$ as $N \rightarrow \infty$.

The result follows immediately by letting $\gamma=\frac{x}{2 \pi}$ in Theorem 5.1 .

Acknowledgments: The authors would like to express their gratitude to Guido Janssen for many detailed comments on an earlier version of the paper. The comments improved the presentation and also lead to much better estimates for some of the Bessel bounds. Furthermore O. C. thanks Say Song Goh for making him aware of the papers [1, 9, 16. The authors thank NIMS for support and hospitality during their visit in February 2017. This research was supported by Basic Science Research Program through the National Research Foundation of Korea(NRF) funded by the Ministry of Education(2016R1D1A1B02009954) and also by National Institute for Mathematical Sciences (NIMS) (A23100000).

\section{References}

[1] Bricks, R.: On the convergence of derivatives of B-splines. Comput. Appl. Math. 27(1), 80-92 (2008)

[2] Casazza, P. G., Christensen, O. and Janssen, A.J.E.M.: Weyl-Heisenberg frames, translation invariant systems, and the Walnut representation. J. Funct. Anal. 180 (2001), $85-147$. 
[3] Christensen, O.: Pairs of dual Gabor frames with compact support and desired frequency localization. Appl. Comput. Harmon. Anal. 20, 403-410 (2006)

[4] Christensen, O.: An introduction to frames and Riesz bases. Second expanded edition. Birkhäuser (2016)

[5] Christensen, O. and Heil, C.: Perturbations of Banach frames and atomic decompositions. Math. Nach. 185 (1997), 33-47.

[6] Christensen, O., Kim, R.Y.: On dual Gabor frame pairs generated by polynomials. J. Fourier Anal. Appl. 16, 1-16 (2010)

[7] Christensen, O., Laugesen, R.: Approximately dual frames in Hilbert spaces and applications to Gabor frames. Sampl. Theory Signal Image Process. 9, 77-90 (2011)

[8] Feichtinger, H.G., Kaiblinger, N.: Varying the time-frequency lattice of Gabor frames. Trans. Amer. Math. Soc. 356, 2001-2023 (2004)

[9] Goh, S.S., Goodman, T.N.T., Lee, S.L.: Appell sequences, continuous wavelet transforms, and series expansions. Appl. Comput. Harmon. Anal. to appear 2016

[10] Janssen, A.J.E.M.: Signal analytical proofs of two basic results on lattice expansions. Appl. Comput. Harmon. Anal. 1, 350-354 (1994)

[11] Janssen, A.J.E.M.: Some Weyl-Heisenberg frame bound calculations. Indag. Math 7 (1996), 165-183.

[12] Lemvig, J., Nielsen, K.H.: Counterexamples to the B-spline conjecture for Gabor frames. J. Fourier Anal. Appl. 22, 1440-1451 (2016)

[13] Lyubarskii, Y.: Frames in the Bargmann space of entire functions. Adv. Soviet Math. 11, 167-180 (1992)

[14] Seip, K.: Density theorems for sampling and interpolation in the Bargmann-Fock space I. J. Reine Angew. Math. 429, 91-106 (1992)

[15] Seip, K., Wallsten, R.: Density theorems for sampling and interpolation in the Bargmann-Fock space II. J. Reine Angew. Math. 429, 107-113 (1992)

[16] Unser, M., Aldroubi, A., Eden, M.: On the asymptotic convergence of B-spline wavelets to Gabor functions. IEEE Trans. Inform. Theory 38(2), 864-872 (1992)

Ole Christensen

Department of Applied Mathematics and Computer Science

Technical University of Denmark, Building 303, 2800 Lyngby

Denmark, Email: ochr@dtu.dk

Hong Oh Kim

Department of Mathematical Sciences, UNIST 
50 UNIST-gil, Ulsan 44919

Republic of Korea, Email: hkim2031@unist.ac.kr

Rae Young Kim

Department of Mathematics, Yeungnam University

280 Daehak-Ro, Gyeongsan, Gyeongbuk 38541

Republic of Korea, Email: rykim@ynu.ac.kr 